\title{
Traduire
}

Ine autre perspective sur la tataduction

Revue française de la traduction

$226 \mid 2012$

Face au miroir

\section{Les marques lexicales du français familier dans la traduction polonaise des dialogues romanesques}

Jadwiga Cook

\section{(2) OpenEdition}

\section{Journals}

Édition électronique

URL : http://journals.openedition.org/traduire/162

DOI : $10.4000 /$ traduire.162

ISSN : 2272-9992

Éditeur

Société française des traducteurs

\section{Édition imprimée}

Date de publication : 1 janvier 2012

Pagination : 93-107

ISBN : 039-773X

ISSN : 0395-773X

Référence électronique

Jadwiga Cook, « Les marques lexicales du français familier dans la traduction polonaise des

dialogues romanesques », Traduire [En ligne], 226 | 2012, mis en ligne le 03 février 2014, consulté le 09 octobre 2020. URL : http://journals.openedition.org/traduire/162 ; DOI : https://doi.org/10.4000/ traduire. 162 


\title{
Les marques lexicales du français familier dans la traduction polonaise des dialogues romanesques
}

\author{
Jadwiga Cook
}

1 Les personnages des romans contemporains parlent une langue proche de la conversation réelle, et les registres de la langue non standard apparaissent souvent dans les œuvres littéraires. Les analyses traductologiques, y compris celles portant sur la traduction littéraire, se concentrent donc aussi sur le registre non standard, car les éléments langagiers peuvent constituer un défi en traduction. Les traducteurs l'admettent en indiquant les éléments familiers ou argotiques comme une des difficultés de leur travail (Desmond 2004). Pourtant, le registre familier présent dans les textes romanesques y joue un rôle important. Il donne des informations sur les personnages, leurs origines sociale ou géographique, leurs traits de caractère ou particularités (Berthelot, 2001, p. 205-206). La traduction devant rendre le même effet, le devoir du traducteur est donc de trouver « cet équivalent qui rendra à la fois, outre le contenu manifeste, l'image et la dynamique du texte original, autrement dit son contenu latent, celui qui fait appel à nos affects et non plus seulement à notre raison » (Desmond, 2004, p. 29). Les difficultés possibles sont de deux types : la langue cible peut ne pas disposer d'un registre équivalent ou, s'il existe, ses marques sont sans doute différentes, comme dans le cas du français et du polonais. Le traducteur est donc souvent obligé de sortir des sentiers bat- tus et de chercher ailleurs des solutions pour rendre dans la langue cible le caractère familier des propos des personnages.

2 Les marques du registre familier ${ }^{1}$ qui sont présentes dans les textes romanesques, notamment dans les dialogues, peuvent être de nature diverse. Les écrivains recourent souvent au lexique non standard, aux éléments syntaxiques, ou bien ils montrent des particularités phoniques dans le discours direct ou dans le discours l'accompagnant. Notre analyse propose une description des moyens employés pour restituer le 
vocabulaire familier et argotique du français dans la traduction polonaise des dialogues romanesques. Cette étude a pour objet de montrer comment ces expressions ont été traitées dans la traduction polonaise des œuvres analysées et jusqu'à quel point ce lexique non standard utilisé par les héros des romans a été conservé et par quels moyens.

3 Le corpus analysé est constitué de romans français contemporains choisis pour la diversité des éléments familiers qui y sont utilisés. Les romans ont été traduits par différents traducteurs, ce qui permet de réduire l'action des facteurs idiolectiques et pourrait aider à trouver d'éventuelles tendances dans la traduction du registre familier vers le polonais.

\section{Le lexique familier en traduction}

4 Les éléments lexicaux constituent le groupe le plus fourni parmi toutes les marques du registre familier dans les textes analysés. Pour les repérer, nous nous sommes servis de dictionnaires de la langue française et de la langue familière et argotique. Les exemples les plus nombreux sont fournis par les expressions dotées dans les dictionnaires du qualificatif familier (370 mots et 40 phraséologismes), ensuite les argotismes (45 mots), les éléments qualifiés de vulgaires (14 mots) et pour finir 7 expressions dites populaires. Nous avons décidé de traiter ces éléments comme un seul groupe, dénommé ici «le lexique familier " pour deux raisons : d'abord l'observation qu'un élément peut être qualifié différemment selon le dictionnaire, ensuite parce que tous ces éléments remplissent dans les textes romanesques la même fonction de caractérisation des personnages et rendent la conversation entre eux plus proche de la communication réelle.

5 Le lexique analysé n'est pas homogène du point de vue morphologique : les lexèmes simples (bagarre, cogner, pompes, salaud) sont les plus nombreux, certains sont un usage familier d'une expression standard (par ex. arroser ou boite). Un autre groupe conséquent est constitué d'abréviations (apéro, beauf, coloc', indic, mac, psy). Il y a un peu moins de mots dérivés par la suffixation familière utilisant les suffixes typiques pour ce registre de la langue, comme -ard (par ex. débrouillard, trouillard, plumard), -os (matos, chicos), -ot (Parigot), -ille (bordille). Nous avons repéré quelques mots composés d'un verbe et d'un substantif (pisse-froid, casse-couilles, fouille-merde). On peut aussi observer des entités provenant de langues étrangères: de l'anglais (shooter, show-biz) ou de l'arabe (toubib, chouffer) et du verlan (chetron, keuf, keum). Il y a aussi un groupe assez fourni de phraséologies familières, comme se faire la malle, mettre un pain à quelqu'un.

Compte tenu du nombre important d'éléments lexicaux observés dans les dialogues analysés, il s'est avéré nécessaire de diviser ce groupe en sous-domaines selon un critère sémantique. Nous avons ainsi cinq catégories thématiques : l'homme, l'homme et les autres, la vie quotidienne, l'homme et la situation, l'homme et les valeurs ${ }^{2}$. On notera que le noyau de ces catégories sémantiques est l'homme. On peut donc constater que le lexique et la phraséologie du registre familier présents dans les romans puisent dans la qualité la plus typique de ce registre, son anthropocentrisme ${ }^{3}$. Nous voyons bien que certains éléments pourraient relever de plusieurs catégories. Or, nous avons décidé de classer les expressions en tenant compte de leur emploi dans le contexte donné. Nous avons limité la présente analyse au groupe le plus fourni en expressions familières - celui concernant l'homme. 
7 Pour analyser les traductions, nous avons utilisé des dictionnaires de la langue polonaise et des dictionnaires spécialisés consacrés au registre non standard de cette langue. Nous considérons comme familiers les éléments qui sont accompagnés dans les dictionnaires des qualificatifs potoczny, pospolity (on peut considérer les deux comme l'équivalent de familier), pogardliwy (méprisant) et wulgarny (vulgaire). Pour chaque solution choisie par les traducteurs, nous indiquons quel type de qualificatif l'accompagne dans les dictionnaires. Les traductions par le lexique standard ont été marquées SQ (sans qualificatif). Lorsque cela était possible, nous avons donné la traduction littérale en regard des expressions polonaises. Nous avons aussi indiqué la fréquence de la traduction choisie entre parenthèses, pour montrer si le choix des expressions familières était plus fréquent que celui du vocabulaire standard.

\section{Le lexique relatif à l'homme}

Le vocabulaire s'appliquant à l'homme constitue le groupe le plus fourni. Ce sont tout d'abord différentes dénominations de l'homme, des expressions parlant de son apparence, de son âge, de ses activités de base et de son état physique et psychique.

\subsection{Les dénominations de l'homme}

Une partie des diverses dénominations de l'homme est constituée d'un lexique neutre, non-valorisant, portant seulement des traits du registre familier. Le reste, par contre, est constitué d'expressions dévalorisantes, souvent méprisantes ou même insultantes. Ce vocabulaire chargé négativement du point de vue émotionnel porte souvent sur la nationalité, la sexualité ou le comportement de la personne concernée.

\subsubsection{Expressions non-valorisantes}

\begin{tabular}{|l|l|l|l|}
\hline Expression originale & Qualificatif & Traduction polonaise & Qualificatif \\
\hline Gars & fam. & $\begin{array}{l}\text { facet (5) [mec], gość (3) [type], } \\
\text { chłopak [garçon, gars] } \\
\text { ludzie (2) [gens] } \\
\text { osobnik [individu] }\end{array}$ & $\begin{array}{l}\text { pot. } \\
\text { SQ } \\
\text { SQ }\end{array}$ \\
\hline Keum & fam. & $\begin{array}{l}\text { facet (2) [mec] } \\
\text { facet (12) [mec], koleś [copain], } \\
\text { dziad [vieillard], chłopaki (2) [gars], } \\
\text { chłop [mec] } \\
\text { nieszczęśnik [misérable] }\end{array}$ & $\begin{array}{l}\text { pot. } \\
\text { Mec }\end{array}$ \\
\hline Meuf & arg. & $\begin{array}{l}\text { kobita (2) [femme, prononciation } \\
\text { familière] } \\
\text { dziewczyna [fille] }\end{array}$ & pot. \\
\hline
\end{tabular}

10 Pour ce groupe de lexèmes, il semble que les traducteurs n'ont pas eu de problème pour trouver les expressions familières équivalentes. Nous n'avons repéré l'emploi du registre standard du polonais que dans quelques cas isolés.

11 Une fois, le mot gars a été traduit osoba płci żeńskiej [une personne de sexe féminin], ce qui (si l'on n'a pas recours au contexte) pourrait suggérer un changement sémantique important. Ce cas est toutefois particulier, puisque gars concerne ici non un homme 
mais un crapaud - l'animal domestique du héros principal du roman de Fred Vargas. En français, crapaud est un nom masculin et l'animal est traité par son propriétaire comme un mâle. Par contre, en polonais, le mot ropucha est un substantif féminin, l'animal est donc traité dans la traduction comme une femelle. Cela influence aussi la traduction du passage cité, tellement loin du registre familier.

\subsubsection{Expressions racistes et relatives à la nationalité}

\begin{tabular}{|c|c|c|c|}
\hline Expression originale & Qualificatif & Traduction polonaise & Qualificatif \\
\hline Bougnoule & fam. & Arab [Arabe] & $S Q$ \\
\hline Bicot & fam. & wszarz [pouilleux] & pot. \\
\hline \multirow[t]{3}{*}{ Crouille } & \multirow[t]{3}{*}{ pop. } & Arab (3) [Arabe] & $S Q$ \\
\hline & & kolorowy [coloré] & pot. pogard. \\
\hline & & $\begin{array}{l}\text { arabskie skurwysyny [fils de putain } \\
\text { arabes] }\end{array}$ & wulg. \\
\hline Rebeu & arg. & beur & - \\
\hline Niaquoué & arg. & wszarz [pouilleux] & pot. \\
\hline \multirow[t]{2}{*}{ Viet } & \multirow[t]{2}{*}{ fam. } & Żóltek (2) [jaune] & pot. pogard. \\
\hline & & Wietnamka [Vietnamienne] & $S Q$ \\
\hline Espingoin & arg. & $\begin{array}{l}\text { Espingoin } \\
\text { przeklęty Hiszpan [Espagnol maudit] }\end{array}$ & SQ \\
\hline \multirow[t]{2}{*}{ Rital } & \multirow[t]{2}{*}{ fam. } & makaroniarz (10) [macaroni] & pot. \\
\hline & & $\begin{array}{l}\text { Rital (+ note en bas de page : } \\
\text { obraźliwa nazwa Włocha } \\
\text { [dénomination insultante d'un } \\
\text { Italien]) }\end{array}$ & \\
\hline
\end{tabular}

Les différences culturelles et le caractère différent de l'immigration en France et en Pologne font qu'il est difficile, voire impossible, de trouver des équivalents pour cette catégorie d'expressions. Dans la langue polonaise, les dénominations péjoratives des immigrés venant des pays du nord de l'Afrique n'existent pas. Les traducteurs ont donc essayé de rendre le caractère injurieux et non standard des expressions à l'aide du vocabulaire péjoratif polonais, comme wszarz ou kolorowy. Il faut quand même souligner que ces dénominations ne font penser à aucune race ou nationalité précise. Une autre solution était d'employer une construction avec un lexème vulgaire. Dans le cas du mot rebeu (qui n'est d'ailleurs pas une expression raciste, mais la forme en verlan du mot beur, employé par les jeunes Arabes eux-mêmes en France), le traducteur a recours à l'emprunt. Ce qui est intéressant, c'est qu'il a décidé d'employer la forme beur et non rebeu. Cela est dû probablement au fait que le mot beur est déjà apparu dans le texte et a été expliqué en note de bas de page et que le traducteur ne voulait pas introduire une autre expression étrangère. 


\subsubsection{Expressions relatives à la sexualité}

\begin{tabular}{|l|l|l|l|}
\hline Expression originale & Qualificatif & Traduction polonaise & Qualificatif \\
\hline \multirow{2}{*}{ Pédé } & \multirow{2}{*}{ fam. } & pedał (7) [pédé] & wulg. \\
\cline { 3 - 4 } & & pedzio [pédé], ciota [tapette] & pot. \\
\hline Tantouse & fam. & pijawka [sangsue] & - \\
\hline
\end{tabular}

La langue polonaise offre des équivalents non standard qui pourraient servir à la traduction du vocabulaire de ce domaine. Il convient toutefois de remarquer que le mot pedat, équivalent de pédé est classé différemment dans les deux langues : en polonais, il est qualifié comme vulgaire tandis qu'en français, il ressort du lexique familier. Dans un cas, on peut supposer qu'il y a eu une erreur dans la traduction. Le terme pijawka (une sangsue) peut être employé comme relatif à une personne, mais seulement pour décrire quelqu'un qui demande souvent de l'argent aux autres, le mot n'est jamais utilisé comme dénomination d'un homosexuel. L'erreur peut être due à la ressemblance entre les mots français tantouse et ventouse.

\subsubsection{Expressions relatives au comportement}

\begin{tabular}{|l|l|l|l|}
\hline Expression originale & Qualificatif & Traduction polonaise & Qualificatif \\
\hline Fumier & fam. & świnia [cochon] & pot. \\
\hline Salaud & $\begin{array}{l}\text { pop. (DFC), } \\
\text { fam. }\end{array}$ & sukinkot, skurwiel [fils de putain] & wulg. \\
\cline { 4 - 4 } & & (stary) drań (2) [(vieux) gredin] & pot. \\
\cline { 3 - 4 } & & świnia (2) [cochon] & pogard. \\
\cline { 3 - 4 } & $\begin{array}{l}\text { szelma [coquin], lajdak (2) [salaud], } \\
\text { tobuz [polisson] }\end{array}$ & SQ \\
\hline
\end{tabular}

Dans ce groupe aussi, les traducteurs ont pu employer souvent le vocabulaire familier ou vulgaire polonais. Le mot świnia (cochon) attire l'attention puisqu'il est qualifié de différentes façons : Doroszewski le décrit comme pogardliwy (méprisant) et Anusiewicz et Skawiński comme potoczny (familier). Comme le lexème apparaît dans des textes publiés à des époques différentes (Bonjour tristesse en 1956 et Total Khéops en 2005), nous indiquons les deux qualificatifs.

\subsection{Vocabulaire relatif à l'apparence physique}

Cette catégorie de lexèmes regroupe d'un côté des expressions décrivant les parties du corps humain, et de l'autre côté, le vocabulaire concernant l'apparence physique d'une personne donnée. 


\begin{tabular}{|l|l|l|l|}
\hline Expression originale & Qualificatif & Traduction polonaise & Qualificatif \\
\hline $\begin{array}{l}\text { Chetron (entrer des trucs dans la } \\
\text { chetron) }\end{array}$ & arg. & $\begin{array}{l}\text { mózg (prać mózgi) [cerveau (laver } \\
\text { des cerveaux)] }\end{array}$ & SQ \\
\hline Tronche & fam. & gęba [gueule] & pot. \\
\cline { 3 - 4 } & Qum. & kości [os] & - \\
\hline Quilles & fam. & $\begin{array}{l}\text { gęba (9) [gueule], pysk [museau], } \\
\text { morda [gueule], teb [caboche] }\end{array}$ & pot. \\
\cline { 3 - 5 } & & $\begin{array}{l}\text { twarz (2) [visage], kark [nuque], oczy } \\
\text { [yeux], mina [mine] }\end{array}$ & SQ \\
\hline Baraqué & barczysty [large d'épaules] & SQ \\
\hline Maigrichon & fam. & chudzielec (2) [maigrichon] & pot. \\
\hline Nippé & fam. & odpicowany [nippé] & pot. \\
\hline
\end{tabular}

16 Comme on peut le voir, les traducteurs n'ont pas toujours recours aux possibilités que donne le polonais familier dans le domaine de la dénomination des parties du corps. Seuls les synonymes non standard des mots głowa (tête) et twarz (visage) ont été employés d'une façon régulière. Pour les autres, les traducteurs ont le plus souvent utilisé des expressions dépourvues de caractère familier. Le mot quilles, par exemple, a été rendu en traduction par une phraséologie połamać kości (casser les os), or il est facile de trouver un équivalent comme kulasy ou giczoły (les deux sont des dénominations non standard des jambes). Il en est de même pour le mot baraqué, pour la traduction duquel on pourrait utiliser un équivalent sémantique familier - napakowany.

\subsection{Expressions décrivant le caractère}

Dans cette catégorie, nous avons regroupé les expressions ayant rapport aux traits de caractère des personnages. 


\begin{tabular}{|c|c|c|c|}
\hline Expression originale & Qualificatif & Traduction polonaise & Qualificatif \\
\hline Bêta & fam. & głuptas [sot] & $S Q$ \\
\hline \multirow[t]{2}{*}{ Con } & \multirow[t]{2}{*}{ fam. } & $\begin{array}{l}\text { idiota (4) [idiot], głupek [niais], } \\
\text { cwany łotr [coquin rusé], zakuty łeb } \\
\text { [tête d'imbécile], pierdoła (3) [con], } \\
\text { osioł [âne], dureń (3) [idiot] }\end{array}$ & pot. \\
\hline & & $\begin{array}{l}\text { głupi (3) [bête], zgłupieć (2) [devenir } \\
\text { bête], nie taki sprytny [pas si malin], } \\
\text { głuptas [sot], niezdara [maladroit] }\end{array}$ & SQ \\
\hline Corniaud & fam. & gburowaty synalek [fils bourru] & $S Q$ \\
\hline Débrouillard & fam. & bystry [malin] & SQ \\
\hline Dinde & fam. & małpa [singe] & pot. \\
\hline \multirow[t]{2}{*}{ Gaillard } & \multirow[t]{2}{*}{ fam. } & zuch chłopak [brave garçon] & pot. \\
\hline & & facet $[\mathrm{mec}]$ & SQ \\
\hline Intello & fam. & $\begin{array}{l}\text { typ intelektualistki } \\
\text { [type d'une intellectuelle] }\end{array}$ & SQ \\
\hline Bordélique & fam. & bałaganiara [bordélique] & pot. \\
\hline Calé & fam. & doświadczony [expérimenté] & SQ \\
\hline \multirow[t]{2}{*}{ Chiant } & \multirow[t]{2}{*}{ fam. } & nudziarz [ennuyant] & pot. \\
\hline & & wkurwiający [chiant], upierdliwy [chiant] & wulg. \\
\hline Cinglé & fam. & stuknięty (2) [cinglé], sfiksowany [cinglé] & pot. \\
\hline Empoté & fam. & nieporadny [maladroit] & $S Q$ \\
\hline
\end{tabular}

La première partie du tableau contient des substantifs relatifs au caractère humain : bêta, con, corniaud ou dinde sont des mots plus ou moins injurieux soulignant un niveau bas d'intelligence (or, cette qualité n'est pas toujours visible dans la traduction, par exemple małpa (singe) renvoie plutôt au comportement de la personne donnée).

D'une manière générale, il n'est pas difficile de trouver en polonais des équivalents sémantiques et stylistiques de ce type d'expressions et en effet, beaucoup parmi eux ont été employés dans la traduction des textes analysés. Débrouillard, gaillard et intello sont des expressions généralement positives mais elles peuvent aussi, selon le contexte, devenir des injures. Cela concerne par exemple le mot intello, employé dans le texte original avec une large dose d'ironie. Cela n'est pas tellement visible dans la traduction polonaise qui est à la fois moins familière et moins ironique.

La deuxième partie du tableau contient des adjectifs renvoyant au caractère des personnages. Ici aussi, on a réussi à trouver des équivalents familiers et, dans deux cas, appartenant au vocabulaire vulgaire.

\subsection{Expressions relatives aux activités physiques}

a) Vocabulaire lié au déplacement et au mouvement 


\begin{tabular}{|l|l|l|l|}
\hline Expression originale & Qualificatif & Traduction polonaise & Qualificatif \\
\hline $\begin{array}{l}\text { Se barrer, se casser, se sauver, } \\
\text { se tailler, se tirer, } \\
\text { foutre le camp, se faire la malle }\end{array}$ & fam. & $\begin{array}{l}\text { spadać (4) [tomber], wynosić się } \\
\text { [sens de : foutre le camp] (3), } \\
\text { zmywać się (2) [sens de : se casser], } \\
\text { zabierać się (2) [se prendre, sens } \\
\text { de : se tirer], zmykać [filer], dać } \\
\text { nogę [donner une jambe, sens de : } \\
\text { se sauver], dać dyla [sens de : se } \\
\text { sauver], ulotnić się [s'échapper], } \\
\text { wiać [venter, sens de : fuir] }\end{array}$ & pot. \\
\cline { 4 - 6 } & & $\begin{array}{l}\text { uciekać (5) [fuir], odejść [partir], } \\
\text { iść [marcher], wycofać się [se retirer], } \\
\text { przygotowywać się do wyjazdu [se } \\
\text { préparer pour le départ], zbiera mu } \\
\text { się na włóczęgę [il sent le besoin de } \\
\text { vagabonder] }\end{array}$ & \\
\hline Débarquer & & przyjeżdżać [arriver (en véhicule)] & SQ \\
\hline Godailler & & szwendać się [flâner] & pot. \\
\hline
\end{tabular}

b) Actions physiologiques

\begin{tabular}{|l|l|l|l|}
\hline Expression originale & Qualificatif & Traduction polonaise & Qualificatif \\
\hline Chier & vulg. & zesrać się, srać (2), nasrać [chier] & wulg. \\
\hline Pisser & fam. & sikać (2), wysikać się, nasikać [pisser] & posp. \\
\cline { 3 - 4 } & & odlać się (2) [sens de : pisser] & wulg. \\
\hline
\end{tabular}

c) Autres

\begin{tabular}{|l|l|l|l|}
\hline Expression originale & Qualificatif & Traduction polonaise & Qualificatif \\
\hline \multirow{2}{*}{ Bouffer } & fam. & żreć (4), zeżreć (4) [dévorer] & pot. \\
\cline { 3 - 4 } & & $\begin{array}{l}\text { gryźć [mordre], jeść (6) [manger], } \\
\text { pożreć [dévorer], obgryźć [mordre], } \\
\text { ogryźć [mordre], wyjeść [manger] }\end{array}$ & SQ \\
\hline Brailler & fam. & paplać [papoter] & pot. \\
\hline Chialer & fam. & beczeć [bêler] & pot. \\
\cline { 3 - 5 } & & $\begin{array}{l}\text { plakać [pleurer], rozplakać się } \\
\text { [se mettre à pleurer] }\end{array}$ & SQ \\
\hline Jacter & gadać [jacter] & pot. \\
\hline Se fringuer & fam. & ubierać się [s'habiller] & SQ \\
\hline Se pieuter & fam. & iść do wyra [aller au pieu] & pot. \\
\hline Roupiller & pop. & drzemać [somnoler] & SQ \\
\hline
\end{tabular}

21 La traduction du verbe bouffer mérite un peu plus d'attention. Comme la langue polonaise dispose d'un large éventail d'expressions familières relatives à cette activité quotidienne (par exemple żré́, szamać wcinać), il semble surprenant que seul l'un d'entre eux ait été utilisé lors de la traduction. Les traducteurs ont eu recours surtout aux verbes appartenant au registre standard. Il faut quand même remarquer que le contexte rend parfois impossible l'emploi d'un élément familier. Quelquefois dans le texte original le verbe bouffer a été utilisé pour décrire une action réalisée par un chien 
et c'est pourquoi nous trouvons dans la traduction des verbes comme gryźć (mordre) ou obgryźć. Dans quelques cas, le verbe en question a été traduit d'une manière plus descriptive en raison du contexte : bouffer ensemble est devenu być razem na kolacji (être ensemble au dîner), emmener bouffer au resto a été traduit zabrać na kolacjĘ (emmener au dîner) ou encore aller bouffer a été rendu par pójść coś przekĄsić (aller prendre une bouchée ). Dans ces cas-là, l'apparition d'une forme plus familière dans le texte polonais pourrait être considérée comme une erreur. Enfin, le verbe bouffer apparaît une fois dans un sens figuré (se débarrasser de quelqu'un) et c'est dans le même sens qu'on le retrouve dans la traduction polonaise avec le verbe wyjeść.

Dans la plupart des autres cas, les traducteurs ont bien trouvé les équivalents non standard des noms d'activités.

Nous voudrions regarder de plus près encore un exemple spécifique du verbe foutre qui est un verbe à plusieurs significations selon lesquelles il a été traduit de différentes façons :

\begin{tabular}{|c|c|c|c|c|}
\hline $\begin{array}{c}\text { Expression } \\
\text { originale }\end{array}$ & Qualificatif & Signification & Traduction polonaise & Qualificatif \\
\hline \multirow[t]{4}{*}{ Foutre } & \multirow[t]{4}{*}{ fam. } & faire & $\begin{array}{l}\text { robić (8) [faire], zrobić [faire, aspect } \\
\text { accompli], narobić [faire], wyprawiać (2) } \\
\text { [faire], siać zamęt [semer confusion], } \\
\text { mieć zajęcie [avoir une occupation], } \\
\text { mieć sprawę na głowie [avoir une affaire } \\
\text { sur la tête] (2) }\end{array}$ & $S Q$ \\
\hline & & donner & $\begin{array}{l}\text { dać (spokój) [donner la paix] (2), } \\
\text { przyprawić (o chandrę) [donner le } \\
\text { cafard à quelqu'un] }\end{array}$ & $S Q$ \\
\hline & & \multirow[t]{2}{*}{ mettre } & $\begin{array}{l}\text { wsunąć [glisser] , wrzucić [jeter], } \\
\text { wbić [enfoncer] } \\
\text { podłożyć (ogień) [mettre le feu] } \\
\text { puścić (z dymem) [lâcher avec la } \\
\text { fumée] (2) (pour la traduction de } \\
\text { l'expression foutre le feu) }\end{array}$ & $S Q$ \\
\hline & & & $\begin{array}{l}\text { wpakować [fourrer], wsadzić (2) } \\
\text { [fourrer], powsadzać [fourrer], } \\
\text { wdepnąć (3) [marcher dans quelque } \\
\text { chose], wtrynić [fourrer], włazić [sens } \\
\text { de : grimper, entrer] }\end{array}$ & pot. \\
\hline
\end{tabular}

Comme on peut l'observer sur le tableau ci-dessus, il n'a pas été possible de trouver des équivalents stylistiques pour le verbe foutre dans le sens de faire et de donner. C'est seulement dans le sens de mettre que certaines expressions familières apparaissent dans la traduction polonaise, surtout dans des contextes ou le verbe signifie mettre quelqu'un en prison (verbes verbes wpakować, wsadzić ou powsadzać).

\subsection{Expressions dénotant l'état psychique et physique}

Les expressions relatives à l'état physique sont surtout représentées dans les textes analysés par des mots dénotant l'état élevé de fatigue, comme être crevé, être à cran, être naze, être vanné. Ce groupe de verbes a été traduit en polonais à l'aide de l'équivalent familier być wykończonym ou de locutions appartenant au registre standard de la langue. 
Les autres mots décrivent l'état psychique des personnages (surtout la folie et la peur) et, là aussi, il était possible d'employer le vocabulaire familier polonais.

\begin{tabular}{|l|l|l|l|}
\hline Expression originale & Qualificatif & Traduction polonaise & Qualificatif \\
\hline Dérailler & fam. & szaleć [être fou] & SQ \\
\hline Être à cran & fam. & być wykończonym (2) [être crevé] & pot. \\
\cline { 3 - 5 } $\begin{array}{l}\text { Être crevé } \\
\text { Être naze }\end{array}$ & $\begin{array}{l}\text { u kresu sił [au bout de forces] } \\
\text { fatalnie się czuć [se sentir fatalement] } \\
\text { Être vanné }\end{array}$ & pop. (DFC) śmiertelnie zmęczonym [être \\
fatigué à mourir] & SQ & \\
\hline Mouiller & pop. & robić w gacie [faire dans son froc] & pot. \\
\hline Péter les plombs & loc. fam. & $\begin{array}{l}\text { odbijać (odbiło mu) [rebondir, sens } \\
\text { de : dérailler] }\end{array}$ & pot. \\
\hline
\end{tabular}

L'étude de la traduction des expressions relatives à l'homme a démontré que les deux langues analysées disposent d'un vaste répertoire de vocabulaire non standard. On peut le remarquer par exemple en feuilletant les dictionnaires spécialisés consacrés à ce registre de la langue. En commençant l'analyse, on pourrait donc s'attendre à un niveau élevé des traductions par des équivalents stylistiques des mots donnés. Et c'est dans ce domaine il est vrai que, en comparaison de la syntaxe et de la phonétique non standard, les traducteurs ont le plus souvent réussi à garder le caractère familier des propos des personnages (dans $60 \%$ des cas). Les traducteurs ont souvent puisé dans les possibilités offertes par la langue polonaise et ont utilisé dans la traduction des phraséologies ou des mots familiers. On observe une grande quantité de mots non standard surtout dans le cas de la traduction des dénominations de l'homme, aussi bien non valorisantes qu'injurieuses, des expressions relatives au comportement humain, faisant allusion au peu d'intelligence de quelqu'un.

Comme on a pu le voir ci-dessus, certains mots appartenant en français au registre familier ont été rendus par des expressions polonaises qualifiées de vulgaires. Cette différence ne résulte pas de l'emploi de mots éloignés sémantiquement, mais plutôt du fait que les mêmes éléments relèvent de registres différents dans chaque langue. Il arrive pourtant que le traducteur choisisse d'utiliser un mot plus fort, même si un équivalent familier existe dans la langue cible.

Nous avons pu observer aussi des situations où les traducteurs ont employé moins fréquemment des équivalents non standard, malgré leur présence dans la langue polonaise. Cela concerne surtout le nom des parties du corps humain. Dans certains cas, le contexte a pu jouer un rôle important dans le choix du traducteur. Même si les équivalents existent, il arrive souvent que leurs sphères d'emploi dans les deux langues ne coïncident pas, ce qui rend impossible leur utilisation dans tous les contextes.

Quand le mot français n'a pas d'équivalent en polonais, le défi imposé au traducteur devient d'autant plus difficile. Le plus souvent dans ce type de situation, nous retrouvons dans le texte cible les expressions appartenant au registre standard, comme dans le cas du verbe foutre dans le sens de faire ou de donner. Il arrive rarement que le traducteur décide de compenser cette perte en ajoutant un élément familier là où il ne se trouvait pas dans le texte original, il faut toutefois souligner que ce procédé est employé aussi pour compenser la perte liée aux phénomènes syntaxiques et phonétiques qui ont dû disparaître partiellement dans la traduction. 

fait des différences culturelles. Dans la langue polonaise, on ne trouve pas de vocabulaire péjoratif pour décrire des personnes venant des pays arabes ou des Espagnols. Là où un tel élément apparaissait dans le texte source, les traducteurs ont dû recourir à des dénominations familières qui pourtant ne renvoyaient pas le lecteur à des nationalités concrètes. Dans deux cas, nous observons l'emprunt de l'expression française : beur et Rital. Le dernier exemple est surprenant car en polonais il existe un mot péjoratif pour désigner un Italien (makaroniarz), et ce mot a bien été utilisé plusieurs fois dans la traduction du même texte.

L'élimination de l'élément familier a été observée seulement dans quelques cas isolés (une fois pour le substantif gars et une fois pour tronche). Il est difficile d'en identifier la raison car dans d'autres contextes les mêmes mots ont été traduits à l'aide du vocabulaire non standard.

Les éléments familiers français ont été traduits dans la plupart des cas par des équivalents stylistiques polonais. Même si cela n'était pas possible à chaque fois, l'emploi d'une large quantité de mots et de locutions non standard a rendu les textes polonais stylistiquement proches de leurs originaux français. De plus, c'est le lexique qui a permis aux traducteurs de compenser certaines déperditions dues à la perte d'autres types d'éléments non standard, inexistants dans la langue cible.

\section{BIBLIOGRAPHIE}

\section{Textes analysés}

BAZIN Hervé, Cri de la chouette, éditions du Progrès, Moscou, 1979 ; Wołanie puszczyka,Varsovie, Czytelnik, 1977 (trad. par Krystyna Byczewska).

GAVALDA Anna, Ensemble c'est tout, J'ai lu, Paris, 2007 ; Po prostu razem, Varsovie, Świat KsiĄżki, 2005 (trad. par Hanna Zbonikowska-Bernatowicz).

IZZO Jean-Claude, Total Khéops, Gallimard, Paris, 1995 ; Total Cheops, Varsovie, WAB, 2005 (trad. par Maryna Ochab).

PENNAC Daniel, La petite marchande de prose, Gallimard, Paris, 1989 ; Mała handlarka prozA, Warszawa, Amber, 1997 (trad. par Małgorzata Cebo-Foniok).

SAGAN Françoise, Bonjour tristesse, Julliard, Paris, 1954 ; Witaj smutku, Katowice, Wydawnictwo KsiĄżnica, 2003 (trad. par Anna Gostyńska et Jadwiga OlĘdzka).

SIMENON Georges, L'Amie de Madame Maigret, Presses de la Cité, Paris, 1952 ; Znajoma pani Maigret, Gdańsk, Wydawnictwo L\&L, 2001 (trad. par Irena Szymańska).

VARGAS Fred, Un peu plus loin sur la droite, éditions Vivane Hamy, Paris, 1996 ; Ławka 102, Varsovie, Prószyński i S-ka, 2005 (trad. par Krystyna Szeżyńska-Maćkowiak).

2. Dictionnaires de la langue française 
CARADEC François, N'ayons pas peur des mots. Dictionnaire du français argotique et populaire, Larousse, Paris, 1998 [DAP].

CELLARD Jacques, REY Alain, Dictionnaire du français non conventionnel, Hachette, Paris,1991.

CHARAUDEAU Patrick, MAINGUENEAU Dominique, Dictionnaire d'analyse du discours, Seuil, Paris, 2002.

COLIN Jean-Paul, Dictionnaire de l'argot, Larousse, Paris,1990 [DArg].

DUBOIS Jean et al., Dictionnaire du français contemporain, Larousse, Paris, 1966 [DFC].

DUNETON Claude, Le guide du français familier, Seuil, Paris,1988.

REY-DEBOVE Jacqueline, Rey Alain (dir.), Le Petit Robert, Dictionnaires Le Robert, Paris, 1972 [PR]. REY-DEBOVE Jacqueline, Rey Alain (dir.), Le Nouveau Petit Robert, Dictionnaires Le Robert, Paris, 2001 [NPR].

3. Dictionnaires de la langue polonaise

ANUSIEWICZ Janusz, Skawiński Jacek, Słownik polszczyzny potocznej,, éd. 3, PWN, Varsovie, 2000 [SPP A-S].

CZESZEWSKI Marcin, Słownik polszczyzny potocznej, PWN, Varsovie, 2006 [SPP Cz].

DOROSZEWSKI Witold (dir.), SłownikjĘzyka polskiego, PWN, Varsovie, 1958 [SJP Dor].

DUBISZ Stanisław (dir.), Uniwersalny słownik jĘzyka polskiego, PWN, Varsovie, 2006 [USJP].

LUBAS Władysław (dir.) Słownik polskich leksemów potocznych, t. 1, Wydawnictwo Naukowe DWN, Varsovie, 2001 ; t. 2-4, Wydawnictwo Lexis, Varsovie, 2003-2006 [SPLP].

SZYMCZAK Mieczysław (dir.), Słownik jĘzyka polskiego, PWN, Varsovie, 1998 [SJP Szym].

4. Études

ANTOINE, F., 2004, «Argots et langue familière : quelle représentation en lexicographie bilingue? », in : idem, Argots, langue familière et accents en traduction, Lille, p. 11-23.

BALLARD Michel, Oralité et traduction, Artois Presses Université, Arras, 2001.

BENSOUSSAN Albert, Un problème de traduction : le dialogue dans le roman, [in :] Actes des deuxièmes assises de la traduction littéraire, Actes Sud, Arles, 1986, p. 115-116.

BERTHELOT Francis, Parole et dialogue dans le roman, Nathan, Paris, 2001.

BUTTLER Danuta, Kategorie semantyczne leksyki potocznej, [in :] Z zagadnień słownictwa współczesnego jĘzyka polskiego, Wrocław, Ossolineum, 1978, p. 37-45

DESMOND, W.O., 2004, « Langue verte et verdeur de la langue », in : Antoine F. (dir.), Argots, langue familière et accents en traduction, Lille, p. 25-29.

ŚwiĘcicka Małgorzata, Kreacja dialogu potocznego we wspótczesnej prozie dla młodzieży, Bydgoszcz, 1999.

\section{NOTES}

1. Nous comprenons comme familier le registre utilisé couramment par tous les locuteurs de la langue, dans la langue parlée ordinaire et aussi dans la langue écrite libre. Le terme recouvre les 
phénomènes lexicaux, syntaxiques et phonétiques plus relâchés que ceux de la langue surveillée (Antoine 2004, p. 12).

2. Nous sommes partis de la proposition de Janusz Anusiewicz et Jacek Skawiński, auteurs du dictionnaire thématique du polonais non standard (SPP A-S).

3. ŚwiĘcicka Małgorzata a abouti à des conclusions similaires lors de son analyse des dialogues romanesques dans la littérature de jeunesse (ŚwiĘcicka 1999, p. 123).

\section{AUTEUR}

\section{JADWIGA COOK}

jadwiga.cook@gmail.com

Jadwiga Cook est chargée de cours à l'Institut d'Études romanes de l'Université de Wrocław

(Pologne). Son doctorat concernait les éléments typiques du français familier dans la traduction polonaise des dialogues romanesques. Actuellement, elle travaille sur les phénomènes syntaxiques polonais et français dans une perspective contrastive. 